\title{
Symmetries and Invariant Solutions for the Coagulation of Aerosols
}

\author{
Mingliang Zheng (D)
}

check for

updates

Citation: Zheng, M. Symmetries and Invariant Solutions for the Coagulation of Aerosols. Mathematics 2021, 9, 876. https://doi.org/ $10.3390 /$ math 9080876

Academic Editor: Theodore E. Simos

Received: 11 March 2021

Accepted: 9 April 2021

Published: 16 April 2021

Publisher's Note: MDPI stays neutral with regard to jurisdictional claims in published maps and institutional affiliations.
College of Mechanical \& Electrical Engineering, Taihu University of Wuxi, Wuxi 214064, China; zhmlwxcstu@163.com or zhengml@wxu.edu.cn

\begin{abstract}
The coagulation of aerosol particles plays an important role in the structural morphological changes of suspended particles at any time and in any space. In this study, based on the Smoluchowski equation of population balance, a kinetic model of aerosol coalescence considering Brownian motion collision is established. By applying the developed Lie group method, we derive the allowed infinitesimal symmetries and group-invariant solutions of the integro-differential equation, as well as the exact solution under some special conditions. We also provide detailed steps and a discussion of the properties. The content and results provide an effective analytic solution for the progressive evolution of aerosol particle size considering boundary and initial conditions. This solution reveals the self-conservative phenomena in the process of aerosol coalescence and also provides validation for the numerical algorithms of general dynamics equations.
\end{abstract}

Keywords: aerosols; coagulation; Lie symmetries; invariant solution; self-conservative

\section{Introduction}

Aerosols are an important part of the atmosphere and can affect various physical and chemical reactions, leading to changes in the environment and even direct effects on human health [1]. The dynamic process of aerosols mainly includes agglomeration, condensation (polymerization), evaporation reduction, advection and convection transport, diffusion, sedimentation, and chemical reactions. The coagulation process is the key component that makes the aerosol particle size increase and the quantity concentration decrease. This component also has an important impact on the uniformity and stability of the aerosol system. Therefore, the particle size distribution, coagulation efficiency, and control of the condensation process are important issues in studying the multiphase flow behaviors of aerosols, which are directly related to the radiation intensity and residence time of the aerosols [2]. From a mathematical point of view, the existing model is a nonlinear integrodifferential equation developed from the group equilibrium equation, so determining the approximate solution or the exact solution for aerosol particle size distribution is a very basic task. Many researchers all over the world have carried out scientific calculations and experiments related to aerosol condensation dynamics [3-13]. However, conventional scientific calculations have some faults (such as numerical errors and the inability to carry out continuous quantitative factor analysis) and no single algorithm is generally applicable. The relevant experiments are also time-consuming and labor-intensive. As described in [14], the Lie group analysis proposed by Sophie Lie is very effective for obtaining an analytic solution of differential equations. For linear or nonlinear equations, ordinary differential equations, and partial differential equations, the algorithm steps of Lie group analysis are fixed and unified, making this method suitable for large-scale computer programming. Therefore, Lie group analysis is also applicable to aerosol dynamics.

The application of Lie group analysis to research on multiphase hydrodynamics has made some progress. For example, the influence of fluid viscosity and the deposition of thermophoretic particles on free heat and mass transfer under different flow conditions was studied using Lie group transformation in [15]. Further, using Lie group analysis, 
the conserved quantity and changes in the velocity and temperature field were obtained for the boundary layer problem of fractional viscoelastic fluid in [16]. These results prove that Lie group analysis is the best method besides numerical simulation. On the one hand, Lie group analysis enables complex problems in hydrodynamics to be solved creatively; on the other hand, this analysis promotes the further development of the Lie group's theory. However, Lie group analysis has not been used to study the condensation behaviors of aerosols. In this paper, considering the dynamic process of aerosol condensation and using the Lie group analysis algorithm, we develop some Lie symmetries and group-invariant solutions for the kinetic equations of aerosol condensation and obtain an accurate analytical general solution expression of particle size distribution, as well as explaining the relevant properties of the solution.

\section{Brownian Condensation Dynamic Equation for Aerosols}

The size distribution of aerosol particles determines the degree of the aerosol's influence. Coagulation is an important physical process in particle growth, as shown in Figure 1. This figure illustrates how the aerosol particles and coagulant rapidly undergo electric neutralization/double-electric layer compression destabilization and then coagulate into a micro-flocculent. The general dynamic equation (with collision efficiency of $100 \%$ ) of the size distribution function of aerosol particles only considering condensation is as follows [3]:

$$
\frac{\partial \bar{n}(\bar{v}, \bar{t})}{\partial \bar{t}}=\frac{1}{2} \int_{0}^{\bar{v}} \bar{\beta}\left(\bar{v}-\bar{v}_{1}, \bar{v}_{1}\right) \bar{n}\left(\bar{v}-\bar{v}_{1}, \bar{t}\right) \bar{n}\left(\bar{v}_{1}, \bar{t}\right) d \bar{v}_{1}-\bar{n}(\bar{v}, \bar{t}) \int_{0}^{\infty} \bar{\beta}\left(\bar{v}, \bar{v}_{1}\right) \bar{n}\left(\bar{v}_{1}, \bar{t}\right) d \bar{v}_{1}
$$

where $\bar{n}(\bar{v}, \bar{t})$ is the particle size distribution function with time $\bar{t}$ and volume $\bar{v}$, and $\bar{\beta}$ is the collision frequency function.

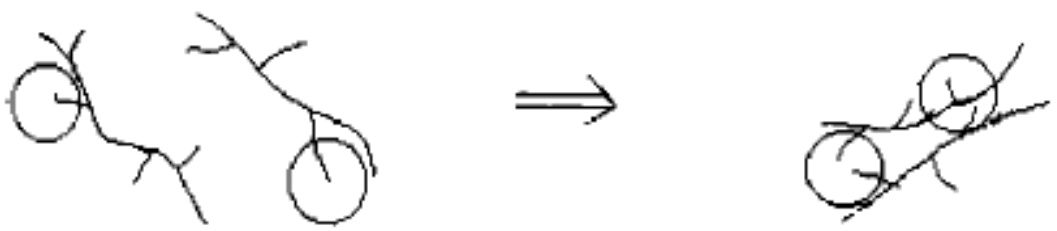

Figure 1. Physical model of the coagulation process.

The collision mechanism of aerosol particles mainly features Brownian motion, shear collision, and differential sedimentation. Brownian motion plays a leading role in collision for systems with smaller particle sizes and higher particle concentrations. The frequency function of monomer collision caused by Brownian motion is expressed in the continuous medium area as [17]

$$
\bar{\beta}_{\text {Brown }}\left(\bar{v}_{i}, \bar{v}_{j}\right)=\frac{2 k_{B} T}{3 \mu}\left(\bar{v}_{i}^{1 / 3}+\bar{v}_{j}^{1 / 3}\right)\left(\bar{v}_{i}^{-1 / 3}+\bar{v}_{j}^{-1 / 3}\right)
$$

where $k_{B}$ is the Boltzmann constant, $T$ is the gas temperature, and $\mu$ is the dynamic viscosity coefficient.

There is a coefficient difference only between the collision frequency function of aerosol particles in the free molecular region and that in the continuous medium region; at the same time, the structure of the initial spherical aggregates will change, and a phenotypic phenomenon will occur in the free molecular region. Thus, this paper does not discuss the free molecular region or transition region. Equation (2) features an important property for the nonzero constant $a$ :

$$
\bar{\beta}_{\text {Brown }}\left(a \bar{v}_{i}, a \bar{v}_{j}\right)=\bar{\beta}_{\text {Brown }}\left(\bar{v}_{i}, \bar{v}_{j}\right) .
$$




\section{Lie Symmetry of the Dynamic Equation of Condensation}

Firstly, the dimensionless transformation of Equation (1) is carried out:

$$
\begin{gathered}
\bar{v}=v_{0} v, \bar{t}=t_{0} t, \bar{n}(\bar{v}, \bar{t})=n_{0} n(v, t), \\
\bar{v}_{1}=\bar{y}=\bar{v} s=v_{0} v s=v_{0} y, \bar{\beta}(\bar{v}, \bar{y})=\beta_{0} \beta(v, y)
\end{gathered}
$$

where $v_{0}, t_{0}, n_{0}, \beta_{0}$ are all positive real numbers.

Using Formula (4), Equation (1) is transformed into

$$
\begin{gathered}
\frac{n_{0} \partial n(v, t)}{t_{0} \partial t}=\frac{1}{2} v_{0} v \int_{0}^{1} \beta_{0} \beta(v(1-s), v s) \cdot n_{0} n(v(1-s), t) \cdot n_{0} n(v s, t) d s \\
-n_{0} n(v, t) \int_{0}^{\infty} \beta_{0} \beta(v, y) \cdot n_{0} n(y, t) d v_{0} y
\end{gathered}
$$

Then, taking $n_{0}=\frac{1}{v_{0} t_{0} \beta_{0}}$, Equation (5) is transformed into

$$
\begin{gathered}
\frac{\partial n(v, t)}{\partial t}=\frac{1}{2} t_{0} n_{0} \beta_{0} v_{0} v \int_{0}^{1} \beta(v(1-s), v s) \cdot n(v(1-s), t) \cdot n(v s, t) d s \\
-t_{0} n_{0} \beta_{0} v_{0} n(v, t) \int_{0}^{\infty} \beta(v, y) \cdot n(y, t) d y \\
=\frac{1}{2} v \int_{0}^{1} \beta(v(1-s), v s) \cdot n(v(1-s), t) \cdot n(v s, t) d s-n(v, t) \int_{0}^{\infty} \beta(v, y) \cdot n(y, t) d y
\end{gathered}
$$

We next introduce a transformation group with a single parameter $\varepsilon$ :

$$
v^{*}=v+\varepsilon \xi_{1}(t, v, n), t^{*}=t+\varepsilon \xi_{2}(t, v, n), n^{*}=n+\varepsilon \eta(t, v, n) .
$$

Using the second-order extension of the Lie group, we can obtain the integral generator of Formula (7):

$$
\phi(t, v, n)=\eta(t, v, n)-\xi_{1}(t, v, n) n_{v}-\xi_{2}(t, v, n) n_{t}
$$

According to the algorithm in [18], under transformation (7), Equation (6) retains form invariance, so we obtain

$$
\begin{gathered}
\frac{\partial n^{*}\left(v^{*}, t^{*}\right)}{\partial t^{*}}=\frac{1}{2} v^{*} \int_{0}^{1} \beta^{*}\left(v^{*}(1-s), v^{*} s\right) \cdot n^{*}\left(v^{*}(1-s), t^{*}\right) \cdot n^{*}\left(v^{*} s, t^{*}\right) d s \\
-n^{*}\left(v^{*}, t^{*}\right) \int_{0}^{\infty} \beta^{*}\left(v^{*}, y\right) \cdot n^{*}\left(y, t^{*}\right) d y \\
\Rightarrow \\
{\left[\phi_{t}+\phi_{n} n_{t}-v \int_{0}^{1} \beta(v(1-s), v s) n(v(1-s), t) \phi(v s, t) d s+\right.} \\
\left.\left.\phi \int_{0}^{\infty} \beta(v, y) n(y, t) d y+n \int_{0}^{\infty} \beta(v, y) \phi(y, t) d y\right)\right]\left.\right|_{\text {the }}(6) \text { is established }=0
\end{gathered}
$$

We next obtain the corresponding solution of Equation (9):

$$
\xi_{1}=c_{1} v, \xi_{2}=-\left(c_{1}+c_{2}\right) t+c_{0}, \eta=c_{2} n
$$

where $c_{0}, c_{1}$ and $c_{2}$ are arbitrary constants. We define the commutator operation-i.e., the lie bracket [,] -in the partial differential operator space $X$ constructed by all group generators of Equation (6) and obtain the corresponding Lie algebraic structure of three dimensions, which have the following basement:

$$
X_{1}=\frac{\partial}{\partial t}, X_{2}=v \frac{\partial}{\partial v}-t \frac{\partial}{\partial t}, X_{3}=t \frac{\partial}{\partial t}-n \frac{\partial}{\partial n}
$$

where $X_{1}$ represents the time shift transformation, and $X_{2}$ and $X_{3}$ represent the stretching transformation.

\section{The Invariant Solution of the Dynamic Equation of Condensation}

If $\mathbf{u}=J(\mathbf{x})$ is the invariant solution of the partial differential equation $F^{m}\left(\mathbf{x}, \mathbf{u}, \partial \mathbf{u}, \partial^{2} \mathbf{u}\right)=0$ and results from the infinitesimal generator $X=\xi_{i}(\mathbf{x}, \mathbf{u}) \frac{\partial}{\partial x_{i}}+\eta_{i}(\mathbf{x}, \mathbf{u}) \frac{\partial}{\partial u^{i}}$, then $X(J)=0$. The group-invariant solution of the equation combined with the method of separating variables can reduce the number of independent variables and can then be transformed 
into an ordinary differential equation, which provides a way to obtain the exact solutions of a given partial differential equation [19].

Aerosols with particle sizes less than $1 \mu \mathrm{m}$ can be regarded as a single spherical dispersion system. Furthermore, the collision frequency response function can be reduced to

$$
\bar{\beta}=\frac{8 k_{B} T}{3 \mu} .
$$

In this way, Equation (6) for aerosol particles in a single dispersion system under the action of single Brownian motion becomes

$$
\frac{\partial n(v, t)}{\partial t}=\frac{1}{2} v \int_{0}^{1} n(v(1-s), t) n(v s, t) d s-n(v, t) \int_{0}^{\infty} n(y, t) d y,\left(n_{0}=\frac{3 \mu}{t_{0} v_{0} 8 k_{B} T}\right) .
$$

For the generator $X_{2}+2 X_{3}$, the invariant is

$$
v \frac{\partial J}{\partial v}+t \frac{\partial J}{\partial t}-2 n \frac{\partial J}{\partial n}=0, \lambda_{1}=v / t, \lambda_{2}=t^{2} n
$$

In this way, we can obtain the general form of the invariant solution of Equation (13):

$$
n(v, t)=t^{-2} f\left(\lambda_{1}\right) .
$$

Substituting Formula (15) into Equation (13), we obtain

$$
\lambda_{1} f^{\prime}+\frac{1}{2} \lambda_{1} \int_{0}^{1} f\left(\lambda_{1}(1-s)\right) f\left(\lambda_{1} s\right) d s+\left(2-\int_{0}^{\infty} f\left(\lambda_{1}\right) d \lambda_{1}\right) f=0 .
$$

Thus, the specific forms of the group-invariant solution and explicit solution of the coagulation dynamic equation are

$$
f\left(\lambda_{1}\right)=e^{-\frac{1}{2} \lambda_{1}}, n(v, t)=t^{-2} e^{-\frac{v}{2 t}} .
$$

Formula (17) indicates that $\lim _{t \rightarrow+\infty} n(v, t)=0, \lim _{v \rightarrow+\infty} n(v, t)=0$, so solution (17) is asymptotically stable, and the population density distribution function of any particle of sufficient size (mass and volume) will inevitably tend to zero.

Supporting the correctness of the results obtained in this paper, several previous studies $[20,21]$ have also shown that the size distribution of aerosol coagulation flocs possesses self-similarity. That is to say, the size distribution shape does not change with time and occurs without consideration of the initial conditions. After a long enough time period, the size distribution of particles will eventually achieve an exponential distribution, so the final conservative spectrum will have a relatively concentrated particle distribution.

The total number $N(t)$ and total volume $\Phi$ of particles in unit volume are as follows:

$$
\begin{gathered}
N(t)=\int_{0}^{\infty} n(v, t) d v=2 / t \\
\Phi=\int_{0}^{\infty} v n(v, t) d v=4
\end{gathered} .
$$

Then, the dimensionless volume $\zeta$ and the dimensionless density function $\Psi(\zeta)$ are

$$
\zeta=\frac{v N}{\Phi}=v / 2 t, \Psi(\zeta)=n(v, t) \Phi / N^{2}=e^{-\zeta} .
$$

Formula (19) shows that the density function accords with the properties of selfconservation and reduction, which is different from any other method used in the past. We applied the results of the Lie group analysis to demonstrate that the coagulation process of aerosols is self-conservative. Even though the particle collision is complex, the overall particle size distribution in the coagulation process can be predicted. 


\section{Conclusions}

Due to the universality and excellent structural properties of the Lie group, the Lie group analysis method is widely used in engineering, mathematics, and physics. These applications have shown that the Lie group analysis algorithm is very effective and agile. To study aerosol evolution dynamics, we used the developed Lie symmetry method to determine the invariance of the aerosol condensation dynamic process under Brownian action and obtain a three-dimensional Lie algebra structure. Then, we reduced the equation and obtained the invariant solution and analytic exact solution of the original equation. The results show that the size distribution of agglomerated flocs of spherical aerosol particles in a single dispersed-phase system possesses self-similarity. In the model itself, the process is rigorous and widely applicable. This method provides a new way to explain the evolutionary characteristics of aerosol physicochemical processes, which have certain theoretical and practical significance. This method could be further extended to other nonlinear dynamics of aerosols.

Funding: This research received a grant from the Natural Science Foundation of Universities in Jiangsu Province (20KJD460001).

Institutional Review Board Statement: Not applicable for studies not involving humans or animals.

Informed Consent Statement: Not applicable for studies not involving humans.

Data Availability Statement: The study did not report any data.

Conflicts of Interest: The author declares no conflict of interest.

\section{References}

1. Zhang, X. Atmospheric aerosols and their climatic effects in China. Prog. Geosci. 2007, 22, 12-16.

2. Hubbard, J.A.; Omana, M.A.; Cheng, M.D. Aerosol dynamics modeling with chemkin-pro surface-kinetics user-routines. J. Therm. Sci. Eng. Appl. 2020, 12, 1-26. [CrossRef]

3. Chen, Z. Research on Brownian Coagulation of Aerosols via the Method of Moments; Zhejiang University: Hangzhou, China, 2014.

4. Si, X. Asymptotic Behavior of Size Distribution of Aerosol Particles Undergoing Self-Preserving Coagulation; Beijing Jiaotong University: Beijing, China, 2017.

5. Gao, J. Efficient Spectral Method for Nonlinear Aerosol Dynamics Equations; Beijing University of Technology: Beijing, China, 2018.

6. Xu, G.; Wang, J. Research on dynamic model of nonspherical ultrafine particles considering both deposition and coagulation. Acta Sci. Circumstantiae 2018, 38, 2757-2763.

7. Sandu, A.; Borden, C. A framework for The Numerical Treatment of Aerosol Dynamics. Appl. Numer. Math. 2003, 45, 475-495. [CrossRef]

8. Elagarayhi, A. Solution of the Dynamic Equation of Aerosols by means of Maximum Entropy Technique. J. Quant. Spectrosc. Radiat. Transf. 2002, 75, 1-11. [CrossRef]

9. Liang, D.; Guo, Q.; Gong, S. A New Splitting Wavelet Method for Solving the General Aerosol Dynamics Equation. J. Aerosol. Sci. 2008, 39, 467-487. [CrossRef]

10. Meimaroglou, D.; Roussos, A.I.; Kiparissides, C. Part IV: Dynamic evolution of the particle distribution in particulate processes.Acomparative study between Monte Carlo and the generalized method of moments. Chem. Eng. Sci. 2006, 61, 5620-5635. [CrossRef]

11. Duru, P.; Koch, D.L.; Cohen, C. Experimental study of turbulence-induced coalescence in aerosols. Int. J. Multiph. Flow 2007, 33, 987-1005. [CrossRef]

12. Hussein, T.; Hruška, A.; Dohányosová, P.; Džumbová, L.; Hemerka, J.; Kulmala, M.; Smolík, J. Deposition rates on smooth surfaces and coagulation of aerosol particles inside a test chamber. Atmos. Environ. 2008, 43, 905-914. [CrossRef]

13. Sorokin, A.A.; Atesh, A.G. Testing Methods for Calculating Particle Coagulation and Settling in an Aerosol Module. At. Energy 2016, 120, 72-77. [CrossRef]

14. Zheng, M. Quantitative Analysis for the Spread Range of Malignant Tumor Based on Lie Symmetry. Complexity 2020, 5, 1-6.

15. Kandasamy, R.; Mu, H. Lie Group Analysis for the Effect of Temperature Dependent Fluid Viscosity and Thermophoresis particle Deposition on Free Convective Heat and Mass Trans fer in the presence of Variable Stream Conditions. Appl. Math. Mech. 2010, 31, 295-305. (In Chinese) [CrossRef]

16. Pan, M. The Applications of Fractional Differential Equations and Lie Group Methods in Studies of Complex Fluids; University of Science and Technology Beijing: Beijing, China, 2018.

17. Zhang, J. The Solution of Kinetic Equation and the Analysis of Self-Preserving of Coagulation; Xi'an University Of Architecture and Technology: Xi'an, China, 2016. 
18. Grigoriev, Y.N.; Kovalev, V.F.; Meleshko, S.V.; Ibragimov, N.H. Symmetries of Integro-Differential Equations: With Applications in Mechanics and Plasma Physics; Springer: New York, NY, USA, 2010.

19. Bluman, G.W.; Anco, S.C. Symmetry and Integral Method of Differential Equations; Yan, Z., Translator; Science Press: Beijing, China, 2009; pp. 54-67. (In Chinese)

20. Swift, D.L.; Friedlander, S.K. The coagulation of hydrosols by Brownian motion and laminar shear tlow. J. Colloid Sci. 1964, 19, 621-647. [CrossRef]

21. Wang, C.S.; Friedlander, S.K. The self-preserving partical size distribution for coagulation by Brownian motion. J. Colloid Interface Sci. $1965,24,126-132$. 